\title{
Implications du médecin du travail et de l'expert dans la gestion et la dosimétrie d'un incident de contamination : exemple d'une plaie contaminée par le plutonium
}

\author{
J. PIECHOWSKI ${ }^{1}$, B. MENOUX ${ }^{2}$, A. MIELE $^{3}$, L. GRAPPIN $^{3}$, \\ A.-M. GUILLERMIN ${ }^{3}$, R. FOTTORINO ${ }^{4}$, M. RUFFIN ${ }^{4}$
}

(Manuscrit reçu le 29 juillet 2002, accepté le 15 janvier 2003)

RÉSUMÉ Le cas rapporté concerne une plaie contaminée par du plutonium. La prise en charge de cet accident amène le médecin du travail de l'INB à résoudre un certain nombre de questions dans le domaine de la dosimétrie, de la communication et des aspects réglementaires. Les notions de dosimétrie et l'interprétation du risque sont complexes en matière de contamination interne. Les limites réglementaires de dose sont des limites annuelles et il est difficile d'expliquer aux victimes d'un incident de contamination que la réglementation détermine les conséquences sur le maintien de l'aptitude au travail en fonction d'une dose engagée sur cinquante ans. Par ailleurs, les déclarations obligatoires à l'employeur, à l'inspection du travail et à l'organisme national de contrôle en radioprotection provoquent de multiples interventions dans le domaine de l'information. Cette observation permet d'évoquer différents aspects pratiques de gestion médicale et de dosimétrie. Dans le cas présenté, le suivi radiotoxicologique et la thérapeutique sont documentés sur une période de plus de cinq ans.

ABSTRACT Implication of the occupational physician and of the expert in the management and the dosimetry of an accidental contamination.

This case concerns a wound contaminated by plutonium. In managing the consequences of such an incident, the occupational physician of a nuclear installation has to solve various questions in the field of dosimetry, communication and regulation. Dosimetry and risk evaluation of internal contamination are complex. Dose limits are annual limits and it is difficult to explain to the victim that for internal contamination the decision concerning the ability to work in controlled areas is determined on the basis of a committed dose on 50 years. Moreover, the notification of such an incident to the employer, to the labour inspectorate and to the governmental organism of control of radiation protection needs an important involvement in the domain of information. This case allows us to describe various practical aspects of medical management and dosimetry. The follow-up of measurements and therapy is given for a period of more than five years.

\footnotetext{
CEA, 3 I ruc de la Fédération, 75752 Paris Cedex 15, France.

Ancien ingénieur IPSN.

CEA, Service medical du travail, 13108 Saint Paut-lez-Durance, France.

4 CEA, Laboratoire d'analyses de biologie médicale, 13108 Saint Paul-lez-Durance, France.
} 


\section{Observation clinique et aspects réglementaires}

\subsection{L'évènement}

Monsieur R, salarié de l'entreprise $\mathrm{C}$, travaille dans un laboratoire d'analyses et de caractérisation radiochimique d'actinides. Le 21 février 1994 vers 14h20, intervenant dans une boite à gants de dissolution nitrique de pastilles de plutonium (type MOX), il serre un collier sur une tuyauterie souple, le tournevis ripe et blesse l'index de la main gauche au niveau de la troisième phalange. Le service de radioprotection confirme immédiatement la contamination et Monsieur $\mathbf{R}$ est rapidement conduit au bloc de décontamination du service médical du centre.

La contamination de la plaie est confirmée, une perfusion par DTPA ${ }^{5}$ est mise en place environ une heure après l'accident (500 mg passés en une heure environ). L'infirmier de permanence pratique une décontamination cutanée soigneuse et prolongée par lavage aux savons liquides (DEK $819^{6}$, Cétavlon $\left.{ }^{(}{ }^{7}\right)$ et rinçage par une solution de DTPA. L'activité résiduelle en plutonium et américium est estimée par une mesure à la sonde $\mathrm{X}$ (cristal NaI-Tl, rendement $1,8 \%$ ) en utilisant un collimateur au plomb centré sur la plaie. Le nombre de chocs permet d'estimer l'activité à $3000 \mathrm{~Bq}$.

Un recueil des urines est mis en route pour une analyse radiotoxicologique.

L'employeur est informé que l'accident entrânera probablement un dépassement des limites réglementaires, et qu'un certain délai est nécessaire pour établir le niveau de l'incorporation avec une précision acceptable.

On notera les éléments suivants :

- la plaie est punctiforme et ne présente pas de caractère de gravité médicale,

- malgré le percement du gant de la boite, il n'y a pas eu d'inhalation concomitante : pas de montée d'activité atmosphérique dans le local de travail, pas d'activité décelée sur les frottis de mucus nasal de Monsieur R,

- le service de radioprotection a retrouvé sur le tournevis utilisé, une forte contamination d'un mélange d'isotopes de plutonium et d'américium sous forme de composés transférables. L'analyse d'un frottis montre :

- en spectrométrie alpha une activité globale de $42000 \mathrm{~Bq}$ dont :

$$
{ }^{239} \mathrm{Pu}+{ }^{240} \mathrm{Pu}=26 \%,{ }^{238} \mathrm{Pu}=60 \%,{ }^{241} \mathrm{Am}=14 \%,
$$

- en scintillation liquide, une activité en ${ }^{241} \mathrm{Pu}$ de $930000 \mathrm{~Bq}$.

\footnotetext{
5 Acide diéthylène triamine penta-acétique.

DEK 819, Laboratoire GEOSYL, 95310 Saint-Ouen, France

${ }^{7}$ Cétavlon $=$ Cétrimide
} 
Le lendemain, les mesures à la sonde $X$ de la plaie sont inchangées; le traitement par DTPA est poursuivi. Dans l'après-midi les résultats d'analyse d'urines du premier jour (J1) confirment la contamination systémique. Un rendez-vous est pris à l'hôpital d'instruction des armées Laveran à Marseille pour une excision chirurgicale.

L'intervention a lieu le 23 février sous anesthésie loco-régionale et perfusion de DTPA (500 mg). Afin de guider le chirurgien, elle se fait avec l'assistance d'un technicien effectuant les mesures à l'aide de la sonde $\mathrm{X}$ qui a servi aux mesures initiales.

Dans la plaie, le trajet de l'outil s'avère plus profond que prévu, les tissus contus sont réséqués fragment par fragment, placés sur une compresse et comptés à la sonde $X$. Le niveau de l'activité éliminée (mesurée sur la compresse) et la diminution de l'activité de la plaie, mais aussi le souci de la sauvegarde fonctionnelle de la phalange, guident le geste chirurgical et déterminent l'arrêt de l'excision.

\subsection{Le suivi}

Le suivi médical de cette personne va prendre en compte les aspects thérapeutiques, dosimétriques, psychologiques et socioprofessionnels de l'accident.

\subsubsection{Thérapeutique}

Le traitement chélateur par DTPA (500 mg en perfusion d'une heure environ) a été répété tous les jours du 21 février jusqu'au 10 mars $1994(\mathrm{~J}=17)$, deux fois par semaine pendant trois semaines, puis une fois par semaine jusqu'au 24 mars 1994 $(\mathrm{J}=31)$ et enfin une fois toutes les deux semaines jusqu'au 18 mars $1995(\mathrm{~J}=390)$, soit un total de 42 injections $(21 \mathrm{~g})$.

Le patient s'est plaint au bout d'un mois, soit vers le 24 mars 1994, de myalgies diffuses des membres inférieurs sans localisation précise et sans aspect évolutif particulier, faisant suspecter un effet secondaire du traitement lié à des perturbations ioniques. Une « fenêtre thérapeutique », c'est-à-dire une suspension du traitement, est alors décidée. Cet arrêt temporaire (du 26 avril au 14 juin 1994 soit 7 semaines) a permis :

1. de pratiquer des examens biologiques à distance des injections (NFS, créatinine, enzymes hépatiques, ionogramme y compris zinc et cuivre) qui se sont révélés normaux,

2. d'évaluer l'efficacité du traitement chélateur par l'observation de la diminution de l'excrétion urinaire pendant la fenêtre thérapeutique. 
Les myalgies ont persisté malgré la prise de calcium, magnésium et oligoéléments, pour s'estomper (paradoxalement) après la reprise du traitement par le DTPA. Ceci nous amène à ne pas retenir l'idée d'un effet iatrogène du DTPA.

\subsection{2. Évaluation dosimétrique}

D'emblée les premiers résultats de mesures (plaie et urines) ont orienté vers une contamination interne importante, cela sera confirmé dans les premières semaines.

L'examen de la décroissance au niveau de la plaie et le suivi des excréta (urines et selles) ont permis d'envisager les hypothèses permettant de faire l'évaluation dosimétrique. En l'absence de modèle établi par la CIPR $^{8}$ pour les blessures contaminées, il a été fait appel pour la détermination de la dose à une expertise spécifique (cf. $\S 2)$.

\subsubsection{Aspects psychologiques}

Ils ne sont pas les moindres. Tout événement « radioactif », surtout s'il entraîne un dépassement des limites réglementaires suscite une grande émotion. Les interventions multiples et souvent dans l'urgence des différentes personnes ayant à traiter l'événement dans l'entreprise et à l'extérieur (enregistrement, déclaration, communication...) créent un climat de tension et des contraintes fortes. Cela trouble l'objectivité nécessaire pour déterminer et présenter à l'individu concerné le réel niveau de gravité de l'incident. C'est un facteur important d'aggravation de son anxiété.

Il importe d'établir une relation suivie entre le patient et les médecins qui l'entourent : le médecin traitant, le médecin du travail de son entreprise, le médecin de l' $^{\prime} \mathrm{NB}^{9}$ qui assure le suivi radiotoxicologique et la prescription de la thérapeutique de décontamination. Ce dernier doit expliquer la conduite du traitement et la surveillance à chacun des acteurs, et notamment à l'intéressé. La démarche adoptée a été établie par les médecins de l'INB en concertation avec les biologistes et les experts en radiotoxicologie, en tenant compte de l'évolution des résultats de mesure et des données médicales. L'adhésion du patient à chaque étape a nécessité de longs entretiens. Monsieur R s'est plié d'une façon exemplaire à la discipline du recueil des excréta ; sa patience et sa rigueur ont permis un grand nombre d'analyses sur des échantillons représentatifs. Les modifications du rythme des injections de DTPA ont été plus difficiles à faire accepter, tout espacement ou arrêt allant à l'encontre d'une forte demande de sa part et de son

\footnotetext{
8 Commission internationale de protection radiologique.

9 Installation nucléaire de base.
} 
désir « d'élimination ». Son inquiétude a été et reste grande quant aux effets à long terme pour sa santé ; cet aspect continue à être pris en compte dans le cadre du suivi médical.

\subsubsection{Aspects socioprofessionnels}

L'étude des circonstances de l'accident a permis un retour d'expérience conduisant à modifier le poste de travail. Le circuit réfrigérant a été mis hors de la boite à gants et l'utilisation des outils contondants a été limitée.

Dès son accident, Monsieur $\mathrm{R}$ a été affecté à un poste de travail sans risque de contamination. Il s'est beaucoup inquiété de son avenir professionnel. En effet, appartenant à une entreprise comprenant un nombre réduit de salariés et intervenant en zone réglementée, une limitation de ses aptitudes pouvait lui faire craindre une rupture de contrat. La situation a pu être négociée avec son employeur et l'entreprise utilisatrice, ce qui lui a permis de sauvegarder son emploi d'une manière stable.

\section{3. Éléments réglementaires en médecine du travail pour les INB}

Le cas rapporté pose plusieurs problèmes au médecin du travail d'une installation nucléaire de base dans trois domaines de ses missions prévues par la réglementation (Code du travail) :

- I'évaluation de l'exposition interne,

- l'information des personnes concernées,

- les décisions d'aptitude/inaptitude.

\subsubsection{L'évaluation des doses par blessure n'est pas explicitée dans le contexte réglementaire actuel}

- Il n'existe pas de modèle biocinétique «normalisé » pour les plaies contaminées. La CIPR a décidé d'en développer un en relation avec le NCRP ${ }^{10}$. Un groupe de travail a été mis en place en 1998 mais son rapport n'est pas encore terminé. La référence choisie a donc été celle du rapport CEA-R5583 (Piechowski et al., 1992).

- Le médecin du travail doit s'assurer d'avoir en sa possession toutes les informations à propos de la composition isotopique du mélange contaminant et de la nature physico-chimique des produits en cause.

- Dans le cas considéré, le médecin du travail a été amené à conduire un traitement au long cours en plus de celui de la phase d'urgence or, en principe,

\footnotetext{
10 National Council on Radiation Protection and Measurements (USA).
} 
il ne peut réglementairement intervenir que durant cette seule phase. La stratégie thérapeutique, en particulier en ce qui concerne sa durée et le rythme d'administration du DTPA, repose sur un compromis entre le bénéfice dosimétrique et la nécessité de limiter le risque d'effets secondaires dus au DTPA (IAEA, 1996 ; Volf, 1989 ; CEC, DOE \& IPSN, 1995). Le DTPA est un chélateur qui a été largement utilisé en milieu hospitalier mais ce n'est pas un médicament avec autorisation de mise sur le marché.

- En liaison avec les experts, le médecin du travail intègre dans sa démarche les particularités que présente l'exposition interne en matière d'évaluation et d'interprétation des doses : mode de calcul, efficacité du DTPA, engagement de dose sur 50 ans, estimation des risques stochastique et non stochastique.

\subsubsection{L'information concerne tout d'abord la victime}

- La perception d'une contamination interne est en général moins bien vécue que celle d'une exposition externe. Le médecin est alors très sollicité pour des renseignements complémentaires. Il ne peut éviter d'aborder les notions complexes d'engagement d'équivalent de dose à un tissu et de somme d'équivalents de doses engagés pondérés. Cette exposition interne doit être ajoutée à l'exposition externe éventuelle sur la même année calendaire. Il est difficile également pour le médecin d'expliquer de manière simple les modalités de l'estimation du risque cancérogène. Le rôle d'accompagnement du médecin et ses qualités de communication dans l'information à donner sont nécessaires pour limiter le doute et donc l'inquiétude chez la victime.

- Sur le plan administratif, en dehors des organismes experts, les obligations de déclaration à différentes instances, provoquent de multiples interventions. Les demandes pressantes d'information jointes en général à la méconnaissance des notions en dosimétrie interne et du temps nécessaire au calcul de celle-ci sont source de confusion et de difficultés. La méthode présentée permet de situer assez rapidement l'ordre de grandeur dès les premiers résultats urinaires, cependant il est difficile de donner une estimation dosimétrique définitive dans l'immédiat du fait de la nécessité d'apprécier l'efficacité du DTPA et de disposer de suffisamment de mesures pour confirmer la validité du modèle métabolique utilisé pour l'interprétation.

- Cette exposition exceptionnelle, dépassant les limites, devient accident d'exposition au sens accident de travail, puisqu'il y a un dépassement d'au moins 10 fois les limites réglementaires annuelles. En contamination interne, il faut tenir compte de l'engagement de dose sur 50 ans et comptabiliser sur l'année calendaire la dose engagée sur 50 ans. On peut remarquer le décalage entre la réalité de l'exposition qui s'étale sur 50 ans et l'obligation réglementaire de la comptabiliser en une fois sur l'année calendaire de l'incident. 


\subsubsection{Les décisions d'aptitude et d'inaptitude ajoutent à l'inquiétude de l'accidenté le souci du devenir professionnel}

- Dans le cas de dépassement des limites de dose, la législation prévoit la mise en place de la surveillance médicale des travailleurs de catégorie A, quelle que soit l'affectation ultérieure de l'intéressé. L'exposition future doit respecter les dispositions de l'article 14 du décret du 2 octobre 1986 modifié par le décret du 19 septembre 1991. Elle est modulée pour qu'à terme les doses cumulées redeviennent compatibles avec les limites.

- Cette dernière disposition allait hypothéquer définitivement les possibilités professionnelles de ce salarié. Cette contrainte, dans le cadre du contrat entreprise utilisatrice et entreprise intervenante, rendait l'avenir de cet opérateur très incertain du fait de l'activité nucléaire spécialisée de sa société. L'entreprise utilisatrice, en l'embauchant, a pallié ce risque et lui a trouvé un poste sans exposition.

\section{Expertise dosimétrique}

\subsection{Résultats des mesures et modèle d'interprétation}

Les résultats des mesures au niveau de la plaie, dans les urines et dans les selles sont donnés dans le tableau I et représentés dans les figures 1, 2 et 3 . On notera l'efficacité du DTPA sur la courbe d'excrétion urinaire, corroborée par la nette diminution des valeurs lorsque le DTPA est arrêté pendant un peu plus d'un mois à partir du $70^{\mathrm{e}}$ jour après l'incident puis définitivement au-delà du $400^{\mathrm{e}}$ jour.

L'interprétation des cas de blessure par le plutonium, en termes de charge corporelle et de dose, a fait l'objet de descriptions détaillées, et de travaux de modélisation et de validation à partir de cas réels (Jolly et al., 1972 ; Schofield, 1969 ; Schofield et al., 1974 ; Carbaugh et al., 1989). Différentes fonctions d'excrétion urinaire et fécale existent (ICRP, 1988, 1998). L'interprétation des résultats de mesure à l'aide de ces fonctions ne conduit pas à des différences substantielles pour l'évaluation des quantités absorbées. On reste dans les mêmes ordres de grandeur. Pour cette expertise, ce sont les fonctions de Durbin (Durbin, 1972) modifiées pour tenir compte des composantes hépatique et osseuse (Piechowski et al., 1992) qui ont été utilisées.

Le schéma de principe pour les transferts à partir d'une blessure contaminée est donné dans la figure 4. Il est important de remarquer que, par opposition aux modèles pulmonaire et digestif développés par la CIPR, le modèle blessure ne peut pas être paramétré de manière détaillée et univoque. Les deux premiers sont en effet des modèles physiologiques, sans dégât au niveau des structures tissulaires et 


\section{TABI.EAU I}

Activités du plutonium et de l'américium au niveau de la plaie (mesures $X$ locales) et dans les excrétions urinaire et fécale (mesures alpha).

Activity of plutonium and americium in the wound (local $X$ measurement) and in the urinary and fecal excretions (alpha measurement).

\begin{tabular}{|c|c|c|c|c|c|}
\hline What & 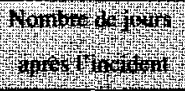 & 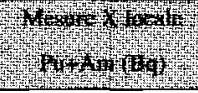 & $\begin{array}{l}44 \\
4 \\
4\end{array}$ & Hot & 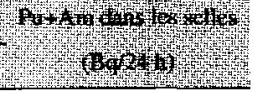 \\
\hline $21 / 2 / 94$ & 0 & 3060 & & & \\
\hline $22 / 2 / 94$ & 1 & 2800 & 59 & & \\
\hline 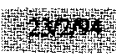 & 14t4ty & 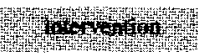 & Ht & H4t & 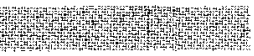 \\
\hline $24 / 2 / 94$ & 3 & & 103 & & \\
\hline $25 / 2 / 94$ & 4 & & 63 & & \\
\hline $26 / 2 / 94$ & 5 & & 76 & & \\
\hline $27 / 2 / 94$ & 6 & & 38 & & \\
\hline $28 / 2 / 94$ & 7 & 600 & 37 & & \\
\hline $1 / 3 / 94$ & 8 & & 29 & & \\
\hline $2 / 3 / 94$ & 9 & & 26 & & \\
\hline $3 / 3 / 94$ & 10 & 550 & 15 & & \\
\hline $4 / 3 / 94$ & 11 & & 19 & & \\
\hline $5 / 3 / 94$ & 12 & & 16 & & \\
\hline $6 / 3 / 94$ & 13 & & 16 & & 0,25 \\
\hline $7 / 3 / 94$ & 14 & 500 & 10 & & \\
\hline $8 / 3 / 94$ & 15 & & 12 & & \\
\hline $9 / 3 / 94$ & 16 & & 11 & & \\
\hline $10 / 3 / 94$ & 17 & 496 & 11 & & \\
\hline $11 / 3 / 94$ & 18 & & 9,7 & & \\
\hline $12 / 3 / 94$ & 19 & & & 2,9 & \\
\hline $13 / 3 / 94$ & 20 & & & 1,9 & \\
\hline $14 / 3 / 94$ & 21 & 496 & & 1,1 & 0,17 \\
\hline $15 / 3 / 94$ & 22 & & 8,4 & & \\
\hline $16 / 3 / 94$ & 23 & & & 2,4 & \\
\hline $17 / 3 / 94$ & 24 & 547 & & 1,6 & \\
\hline $18 / 3 / 94$ & 25 & & 12 & & \\
\hline $19 / 3 / 94$ & 26 & & & 2,2 & \\
\hline $20 / 3 / 94$ & 27 & & & 1,3 & \\
\hline $21 / 3 / 94$ & 28 & 476 & & 1,3 & 0,16 \\
\hline $22 / 3 / 94$ & 29 & & 5 & & \\
\hline $24 / 3 / 94$ & 31 & 434 & & 2 & \\
\hline
\end{tabular}


TABLEAU I (Suite)

\begin{tabular}{|c|c|c|c|c|c|}
\hline \multirow{2}{*}{ part } & \multirow{2}{*}{ 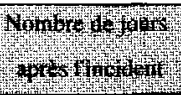 } & \multirow{2}{*}{ 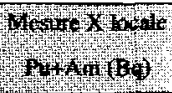 } & \multicolumn{2}{|c|}{ 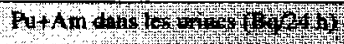 } & \multirow{2}{*}{ 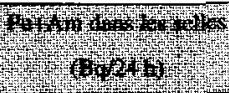 } \\
\hline & & & DTPX & mInth & \\
\hline $25 / 3 / 94$ & 32 & & 13,7 & & \\
\hline $28 / 3 / 94$ & 35 & 432 & & 1,2 & \\
\hline $29 / 3 / 94$ & 36 & & 12 & & \\
\hline $31 / 3 / 94$ & 38 & 461 & & 1,4 & \\
\hline $1 / 4 / 94$ & 39 & & 12,7 & & \\
\hline $5 / 4 / 94$ & 43 & & & 0,75 & \\
\hline $6 / 4 / 94$ & 44 & 410 & 11.7 & & \\
\hline $12 / 4 / 94$ & 50 & 397 & & 0,84 & \\
\hline $13 / 4 / 94$ & 51 & & 12,4 & & \\
\hline $19 / 4 / 94$ & 57 & & & 0,65 & \\
\hline $20 / 4 / 94$ & 58 & & 11,8 & & \\
\hline $26 / 4 / 94$ & 64 & 410 & & 0,6 & \\
\hline $27 / 4 / 94$ & 65 & & 10,9 & & \\
\hline $4 / 5 / 94$ & 72 & & & 1,2 & \\
\hline $11 / 5 / 94$ & 79 & & & 0,5 & \\
\hline $18 / 5 / 94$ & 86 & 282 & & 0,32 & \\
\hline $25 / 5 / 94$ & 93 & 284 & & 0,23 & \\
\hline $31 / 5 / 94$ & 99 & & & 0,22 & 0,071 \\
\hline $1 / 6 / 94$ & 100 & & & 0,15 & \\
\hline $8 / 6 / 94$ & 107 & & & 0,22 & \\
\hline $15 / 6 / 94$ & 114 & 286 & & 0,18 & \\
\hline $16 / 6 / 94$ & 115 & & 9,3 & & \\
\hline $21 / 6 / 94$ & 120 & & & & \\
\hline $22 / 6 / 94$ & 121 & & & 0,47 & 0,78 \\
\hline $23 / 6 / 94$ & 122 & & 7,7 & & \\
\hline $24 / 6 / 94$ & 123 & & & 1,9 & \\
\hline $27 / 6 / 94$ & 126 & 257 & & & \\
\hline $30 / 6 / 94$ & 129 & & & 0,46 & \\
\hline $1 / 7 / 94$ & 130 & & 6,2 & & \\
\hline $7 / 7 / 94$ & 136 & & & 0,45 & \\
\hline $8 / 7 / 94$ & 137 & & 6.25 & & \\
\hline $17 / 7 / 94$ & 146 & & & & 0,47 \\
\hline $18 / 7 / 94$ & 147 & 220 & & & \\
\hline $21 / 7 / 94$ & 150 & & & 0,33 & \\
\hline $22 / 7 / 94$ & 151 & & 4,5 & & \\
\hline
\end{tabular}


J. PIECHOWSKI et al.

TABLEAU I (Suite)

\begin{tabular}{|c|c|c|c|c|}
\hline $4+4+4$ & 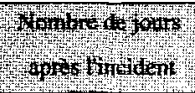 & 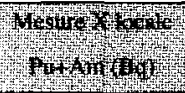 & HA & 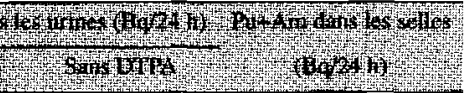 \\
\hline $29 / 7 / 94$ & 158 & 201 & & 0,36 \\
\hline $30 / 7 / 94$ & 159 & & 5,2 & \\
\hline $31 / 7 / 94$ & 160 & & & \\
\hline $16 / 8 / 94$ & 176 & 168 & & 0,19 \\
\hline $17 / 8 / 94$ & 177 & & 4,2 & \\
\hline $31 / 8 / 94$ & 191 & 177 & & \\
\hline $2 / 9 / 94$ & 193 & & & 0,14 \\
\hline $3 / 9 / 94$ & 194 & & 5,3 & \\
\hline $16 / 9 / 94$ & 207 & & & 0,12 \\
\hline $17 / 9 / 94$ & 208 & & 4,8 & \\
\hline $19 / 9 / 94$ & 210 & 157 & & \\
\hline $30 / 9 / 94$ & 221 & 149 & & 0,09 \\
\hline $1 / 10 / 94$ & 222 & & 3,4 & \\
\hline $14 / 10 / 94$ & 235 & & & 0,14 \\
\hline $15 / 10 / 94$ & 236 & & 4 & \\
\hline $17 / 10 / 94$ & 238 & 142 & & \\
\hline $4 / 11 / 94$ & 256 & & & 0,06 \\
\hline $5 / 11 / 94$ & 257 & & 3,3 & \\
\hline $7 / 11 / 94$ & 259 & 130 & & \\
\hline $18 / 11 / 94$ & 270 & 127 & & $0, \mathrm{II}$ \\
\hline $19 / 11 / 94$ & 271 & & 3,4 & \\
\hline $21 / 11 / 94$ & 273 & 126 & & \\
\hline $2 / 12 / 94$ & 284 & 118 & & 0,092 \\
\hline $3 / 12 / 94$ & 285 & & 2,9 & \\
\hline $19 / 12 / 94$ & 301 & 108 & & 0,087 \\
\hline $20 / 12 / 94$ & 302 & & 3 & \\
\hline $12 / 1 / 95$ & 325 & 100 & & \\
\hline $20 / 1 / 95$ & 333 & & & 0,043 \\
\hline $21 / 1 / 95$ & 334 & & 1,9 & \\
\hline $31 / 1 / 95$ & 344 & 108 & & \\
\hline $3 / 2 / 95$ & 347 & & & 0,081 \\
\hline $21 / 2 / 95$ & 365 & 90 & & \\
\hline $21 / 2 / 95$ & 365 & & & 0,045 \\
\hline $22 / 2 / 95$ & 366 & & 2,4 & \\
\hline $17 / 3 / 95$ & 389 & & & 0,032 \\
\hline
\end{tabular}


IMPLICATIONS DU MÉDECIN DU TRAVAIL ET DE L'EXPERT

TABLEAU I (Suite)

\begin{tabular}{|c|c|c|c|c|c|}
\hline \multirow{2}{*}{ Dats } & \multirow{2}{*}{ 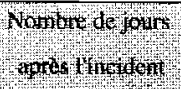 } & \multirow{2}{*}{ 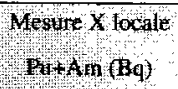 } & \multicolumn{2}{|c|}{ 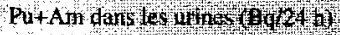 } & \multirow{2}{*}{ 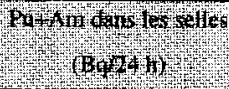 } \\
\hline & & & DTPA & 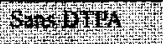 & \\
\hline $18 / 3 / 95$ & 390 & & 1,8 & & \\
\hline $10 / 5 / 95$ & 443 & 80 & & & \\
\hline $15 / 5 / 95$ & 448 & & & 0,026 & \\
\hline $12 / 6 / 95$ & 476 & 84 & & 0,028 & \\
\hline $19 / 7 / 95$ & 513 & 77 & & & \\
\hline $25 / 7 / 95$ & 519 & & & 0,021 & \\
\hline $25 / 8 / 95$ & 550 & 72 & & & \\
\hline $10 / 9 / 95$ & 566 & & & 0,024 & \\
\hline $15 / 9 / 95$ & 571 & 55 & & & \\
\hline $4 / 12 / 95$ & 651 & 53 & & & \\
\hline $6 / 12 / 95$ & 653 & & & 0.012 & \\
\hline $2 / 2 / 96$ & 711 & 57 & & & \\
\hline $4 / 2 / 96$ & 713 & & & 0,021 & \\
\hline $30 / 8 / 96$ & 921 & 59 & & & \\
\hline $3 / 10 / 96$ & 955 & & & 0,021 & \\
\hline $5 / 12 / 96$ & 1018 & & & 0,015 & \\
\hline $2 / 7 / 97$ & 1227 & 44 & & & \\
\hline $4 / 7 / 97$ & 1229 & & & 0.012 & \\
\hline $24 / 10 / 97$ & 1341 & 33 & & & \\
\hline $13 / 11 / 97$ & 1361 & & & 0,011 & \\
\hline $6 / 5 / 98$ & 1535 & 44 & & & \\
\hline $10 / 5 / 98$ & 1539 & & & 0.010 & \\
\hline $16 / 12 / 98$ & 1759 & 42 & & & \\
\hline $26 / 12 / 98$ & 1779 & & & 0,011 & \\
\hline $2 / 3 / 99$ & 1835 & 43 & & & \\
\hline $29 / 2 /(0)$ & 2199 & 42 & & & \\
\hline $13 / 3 / 00$ & 2212 & & & 0,017 & \\
\hline $7 / 2 / 61$ & 2543 & & & 0.013 & \\
\hline $20 / 9 / 0 !$ & 2768 & 35 & & & \\
\hline $27 / 9 / 01$ & 2775 & & & 0.014 & \\
\hline $15 / 5 / 02$ & 2916 & 37 & & & \\
\hline $21 / 5 / 02$ & 3011 & & & 0,006 & \\
\hline $1 / 8 / 02$ & 3083 & 41 & & & \\
\hline $27 / 8 / 02$ & 3109 & & & 0,011 & \\
\hline
\end{tabular}




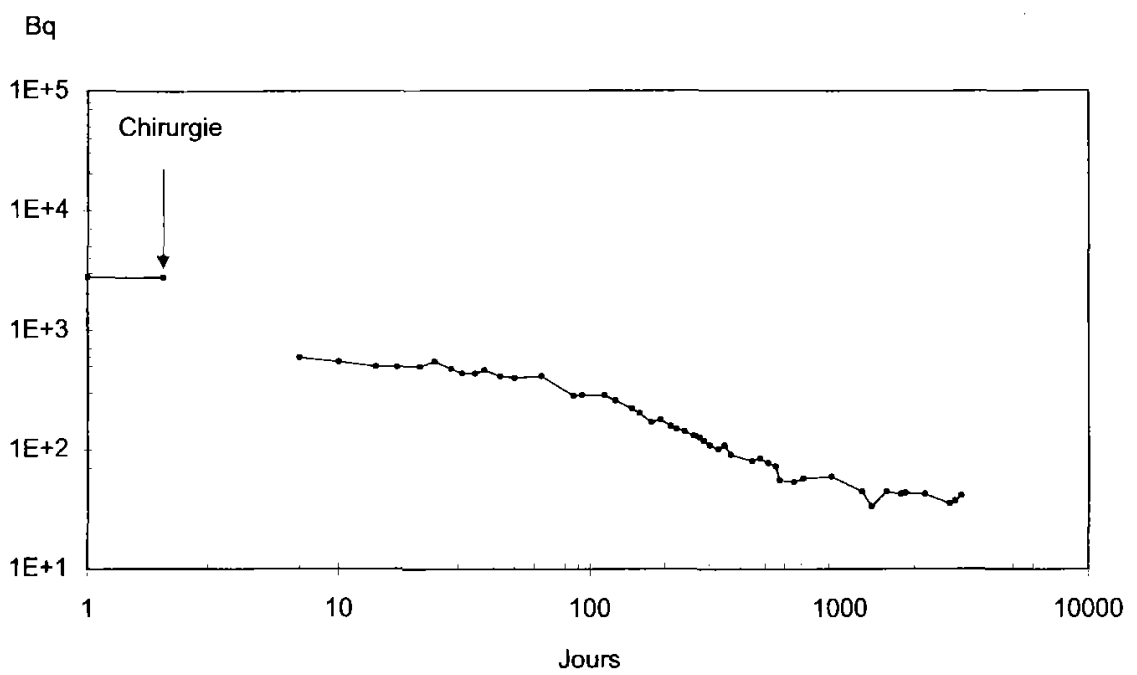

Figure 1 - Mesures locales de l'activité alpha au niveau de la blessure puis de la cicatrice.

Local measurement of alpha activity in the wound and then in the scar.

avec une biocinétique reproductible des traces radioactives qui pénètrent par les voies normales. La blessure, elle, est totalement différente avec des brèches et altérations tissulaires variables d'un cas à l'autre et une entrée des radionucléides par des voies anormales. Le modèle est ici physio-pathologique. Seules des indications générales peuvent être tirées des cas observés, le plus souvent simplement qualitatives. Des situations ectopiques, peu en accord avec le modèle général, ne peuvent être exclues.

En ce qui concerne le plutonium, composé très peu ou pas transférable, une étude réalisée sur plusieurs cas (Piechowski et al., 1989) a montré que, schématiquement, le plutonium ayant pénétré dans la plaie subissait l'évolution suivante :

- une partie entre très rapidement dans le sang par les brèches vasculaires, c'est le transfert systémique relatif à la composante $Q_{2}$; c'est précisément ce plutonium qui peut être mesuré sur de très longues périodes (années) après la blessure,

- l'autre partie, restée au niveau de la plaie, semble inerte ; elle n'est pas ou très peu métabolisée, autrement dit son transfert vers le sang est virtuellement inexistant. Toute cette activité locale, non transférée, correspond à la composante $Q_{1}$. Comme généralement une excision est faite, l'activité 
Bq/jour

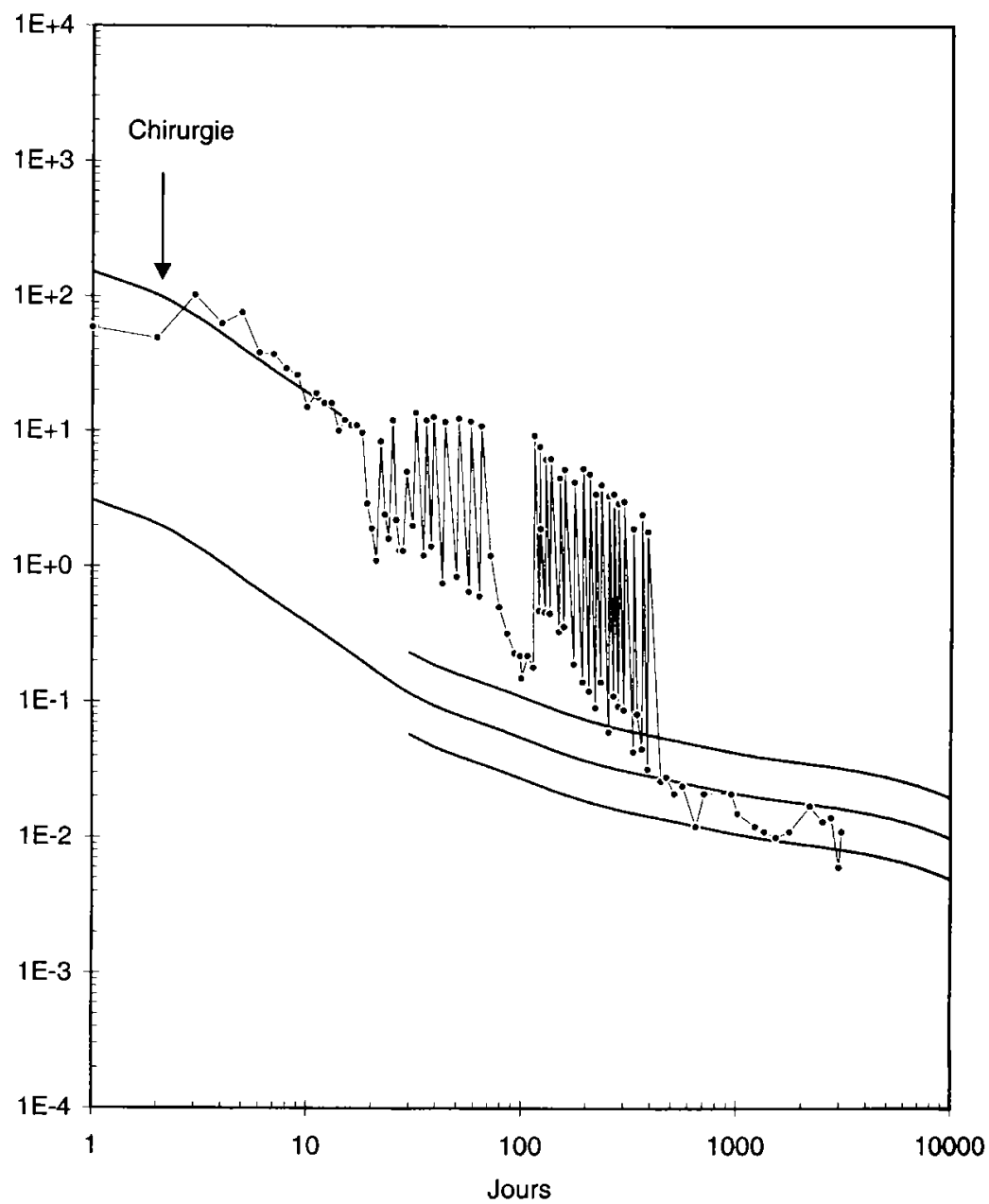

Figure 2-Excrétion urinaire journalière de l'activité alpha; les points supérieurs correspondent à l'excrétion sous DTPA; la courbe continue de I à 10000 jours représente l'excrétion urinaire nominale en l'absence de DTPA.

Daily urinary excretion of alpha activity; the upper points correspond to the excretion after $D T P A$ administration; the unbroken curve from 1 till 10000 days represents the nominal urinary excretion without DTPA. 


\section{Bq/jour}

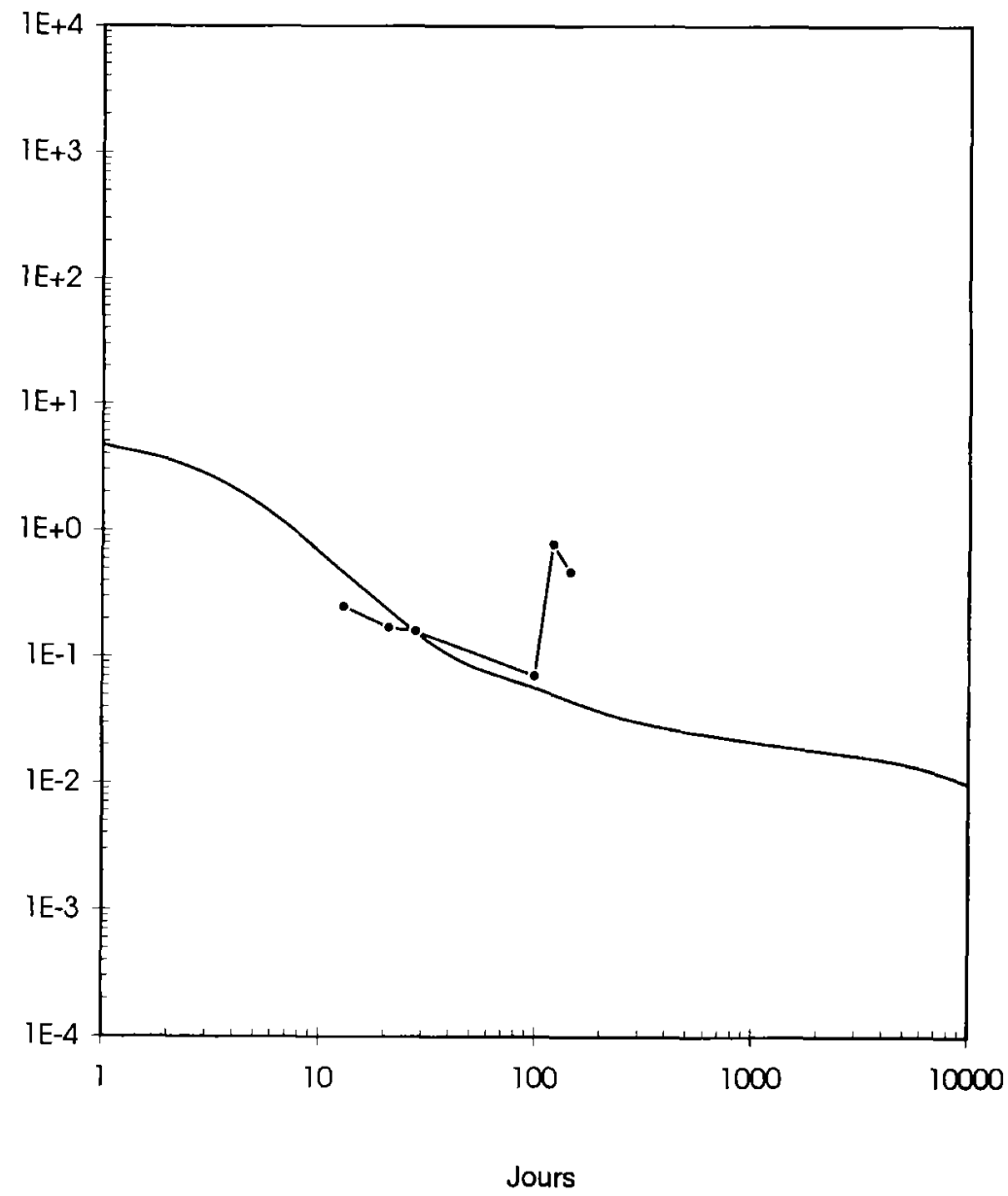

Figure 3-Excrétion fécale journalière de l'activité alpha; la courbe continue de 1 à 10000 jours représente l'excrétion fécale nominale sans DTPA.

Daily fecal excretion of alpha activity; the unbroken curve from 1 till 10000 days represents the nominal fecal excretion without DTPA. 


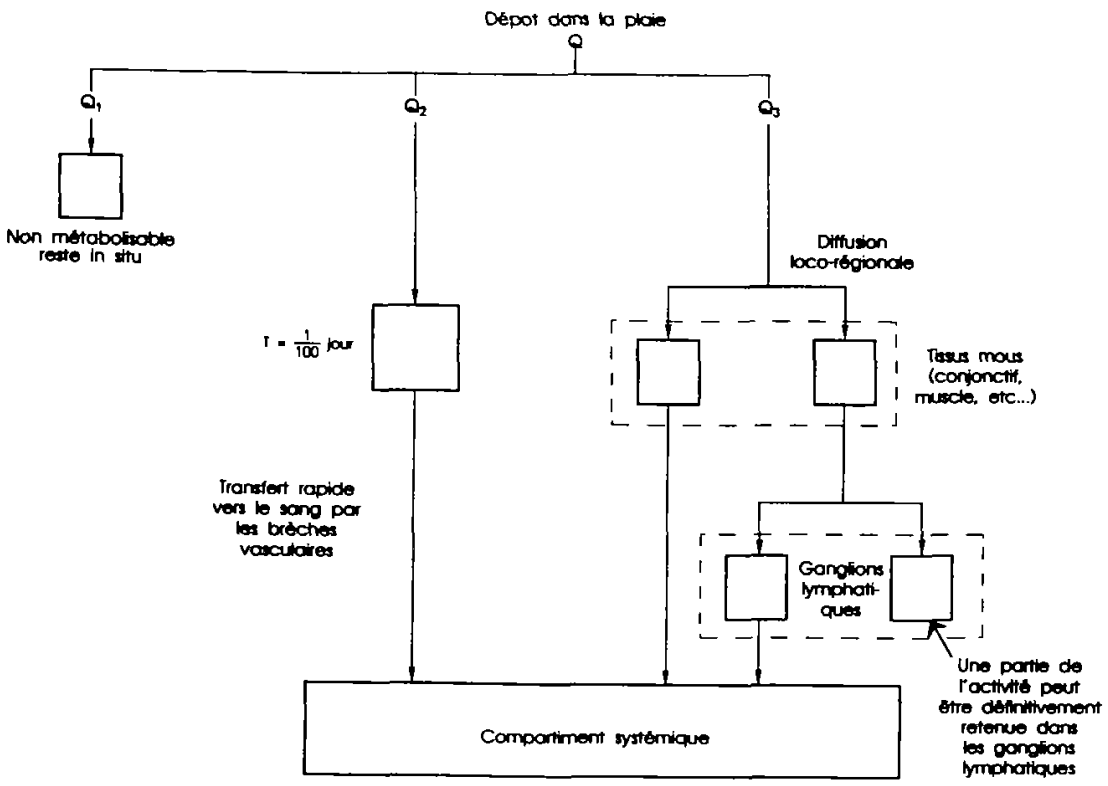

Figure 4 - Schéma des transferts à partir d'une blessure contaminée (Piechowski et al., 1992).

Diagram of the transfers from a contaminated wound (Piechowski et al., 1992).

résiduelle locale, qui subsiste, est $Q_{1, r}$. Dans certains cas, elle peut partiellement s'éliminer lors du renouvellement de l'épithélium cutané,

- en pratique, dans la composante à transfert lent, $Q_{3}$, on observe généralement une migration loco-régionale par voie lymphatique mais les sous-composantes de transfert vers le sang n'existent pas.

Les blessures avec contamination par le plutonium justifient toujours une injection de DTPA effectuée dès que possible. On peut considérer qu'il s'agit d'une urgence médicale. Le but est d'augmenter l'excrétion urinaire de l'activité rapidement absorbée par les brèches vasculaires, correspondant à la composante $Q_{2}$. L'excrétion urinaire sous DTPA est augmentée d'un facteur 25 à 100, 50 en moyenne, par rapport à l'excrétion hors DTPA.

La cinétique d'épuration plasmatique du plutonium sous DTPA peut être caractérisée par deux phases. Dans un premier temps, pendant le laps de temps de l'ordre d'une heure qui précède le traitement par le DTPA, la moitié de l'activité qui a pénétré dans le sang au moment de l'accident quitte la circulation pour se 
répartir dans l'organisme et se fixe principalement dans l'os et le foie. Elle correspond à la première composante de la fonction de rétention plasmatique :

$$
\begin{aligned}
0,524 \mathrm{e}^{-[0,693 / 0,0139] t} & +0,271 \mathrm{e}^{-[0,693 / 0,3042] t}+0,172 \mathrm{e}^{-[0,693 / 1,2] t} \\
& +0,033 \mathrm{e}^{-[0,693 / 5,0] t}+0,0044 \mathrm{e}^{-[0,693 / 88] t}
\end{aligned}
$$

le temps $t$ étant exprimé en jours. Cette fonction résulte d'une étude de cinétique sanguine du ${ }^{239} \mathrm{Pu}$ réalisée chez l'homme. La période de la première composante a une valeur nominale de 20 minutes, avec un intervalle allant de quelques minutes à environ 1 heure (Durbin, 1972). Une autre étude réalisée avec du ${ }^{237} \mathrm{Pu}$ conclue à une épuration initiale moins importante, soit $10 \%$ au lieu de $50 \%$ (Talbot et al., 1993); cette différence de comportement métabolique peut être attribuée à l'impact physico-chimique des propriétés nucléaires des deux isotopes: activités massiques dans un rapport de 200000 (Harrisson et al., 1978) et radioactivité de type capture électronique pour l'un et émission alpha pour l'autre. Dans le cas présent, l'isotope en cause étant le ${ }^{239} \mathrm{Pu}$, nous retiendrons pour l'épuration initiale du compartiment sanguin la fraction \# 0,5 donnée dans la fonction ci-dessus.

Dans la deuxième phase, après mise en place du traitement par le DTPA, l'autre moitié de l'activité, restée dans le sang, est chélatée et excrétée pendant les 2 à 3 semaines suivantes avec une période d'environ 5 jours. Ainsi, la charge systémique qui se produit pendant la phase pré-DTPA est numériquement égale à l'excrétion urinaire cumulée des premières semaines de la phase post-DTPA. Cette dernière peut être évaluée à l'aide de la fonction mono-exponentielle d'excrétion du Pu-DTPA, de période $5 \pm 1$ jours (Schofield et al., 1974), dont on notera que le tracé est virtuellement superposable à celui de la partie initiale de la fonction d'excrétion urinaire du plutonium (Durbin, 1972) multipliée par 50 (Fig. 5). Il est donc possible d'utiliser la courbe de Durbin pour le calcul de la charge systémique en appliquant la règle du facteur 50 pour l'action du DTPA. Cette approche présente l'avantage de la simplicité pour le praticien qui peut, dans tous les cas, utiliser la fonction de Durbin, soit directement pour les résultats d'excrétion urinaire hors DTPA, soit en appliquant le facteur d'efficacité de 50 s'il y a eu administration de DTPA. Si $M$ est un résultat de mesure au temps $t$ après l'incident et $m$ la valeur correspondante de la fonction de Durbin, au même temps $t$, lue sur la courbe d'excrétion théorique (Fig. 6), la charge systémique est estimée par le rapport $\mathrm{M} / \mathrm{m}$ (hors DTPA) ou $\mathrm{M} / 50 \mathrm{~m}$ (sous DTPA). Si l'on dispose de plusieurs résultats successifs, une meilleure estimation est obtenue en prenant la moyenne géométrique des valeurs déterminées à partir de ces mesures. La moyenne géométrique permet de raisonner en ordre de grandeur (échelle logarithmique). 
$\mathrm{Bq}$ /jour

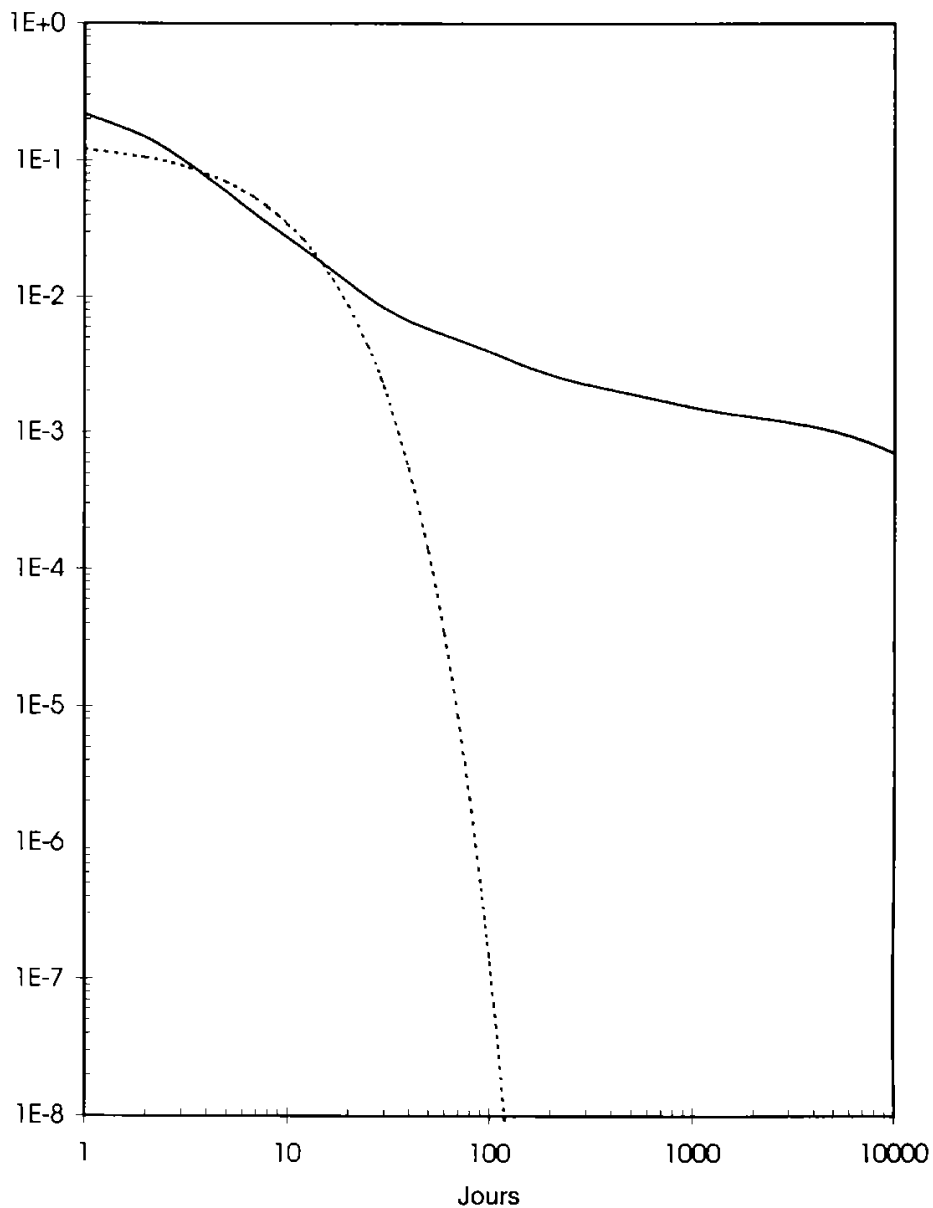

Figure 5 - Comparaison des courbes d'excrétion urinaire du plutonium sous DTPA : la courbe en trait plein représente l'excrétion prévue après administration de DTPA en admettant un facteur d'efficacité de 50 (Durbin, 1972; Piechowski et al., 1992); la courbe en trait pointillé représente l'excrétion du complexe Pu-DTPA (Schofield et al., 1974). On notera la similitude des courbes pendant les 3 premières semaines.

Comparison of the curves of urinary excretion of plutonium after administration of DTPA: the unbroken line corresponds to the expected excretion after administration of DTPA assuming an efficiency factor of 50 (Durbin, 1972; Piechowski et al., 1992); the dotted line represents the excretion of the Pu-DTPA complex (Schofield et al., 1974). Notice the similarity of the curves during the 3 first weeks. 


\section{$\mathrm{Bq} / \mathrm{jour}$}

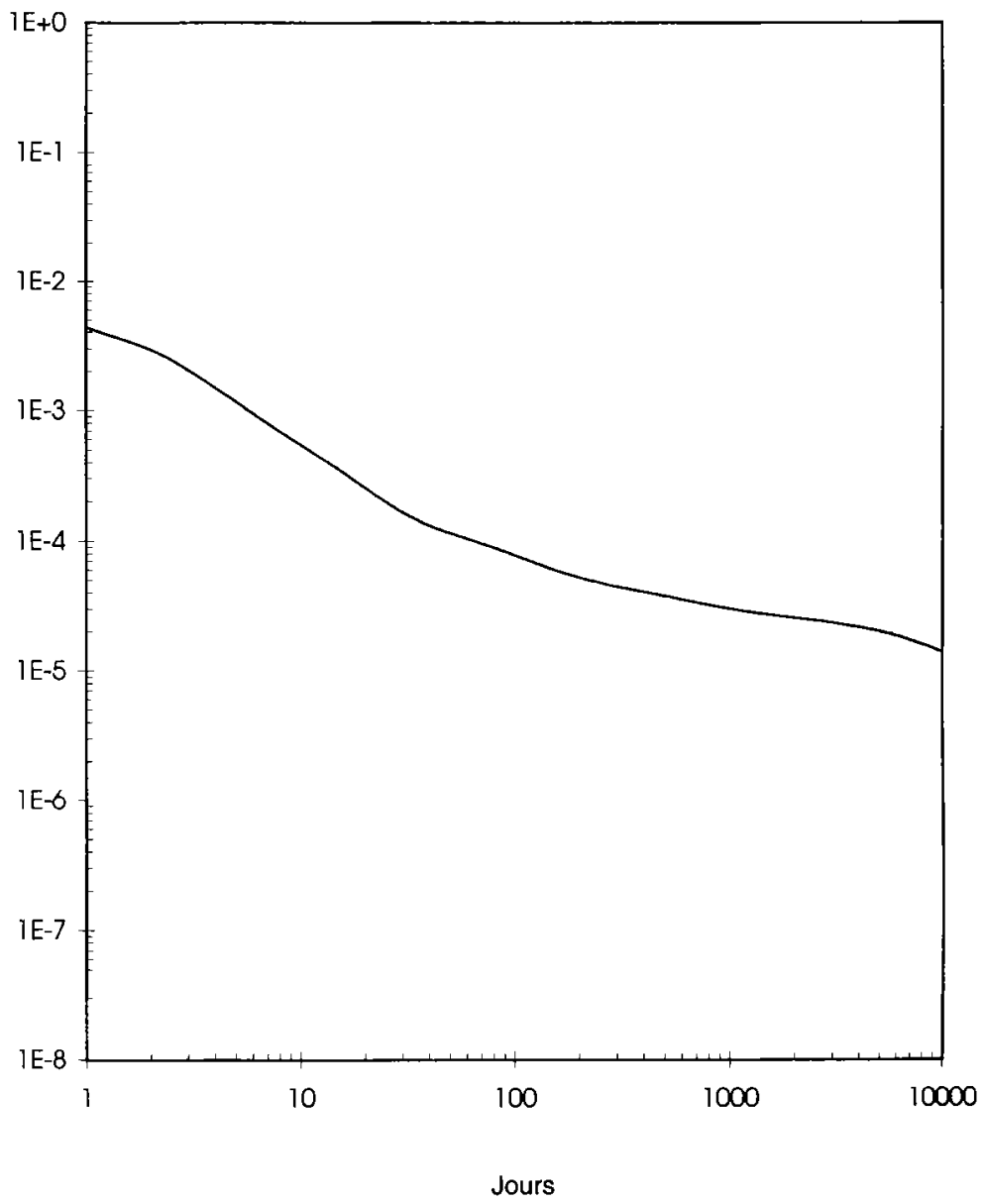

Figure 6-Fonction d'excrétion urinaire du plutonium sans administration de DTPA (Durbin, 1972; Piechowski et al., 1992).

Function of urinary excretion of plutonium without DTPA (Durbin, 1972; Piechowski et al., 1992). 
En ce qui concerne la charge locale au niveau de la blessure, on note une diminution progressive des résultats de mesure (Fig. 1). Cette variation peut s'expliquer par deux composantes :

- une variation «géométrique » due à l'enfouissement et à l'encapsulation dans une fibrose, déterminant une baisse progressive du rendement de comptage et donc l'impression que la charge au niveau de la cicatrice décroît ;

- une variation «biologique » par migration loco-régionale, essentiellement lymphatique et impliquant la participation des cellules phagocytaires; dans ce cas, le produit diffuse régionalement vers les ganglions mais le passage systémique vers le sang reste négligeable.

Selon les blessures et la nature du produit, ces deux composantes sont plus ou moins importantes, de même qu'elles sont plus ou moins associées. Il n'y a pas de règle modélisable en la matière. Ces situations ont été décrites et vérifiées (Bistline et al., 1972 ; Hammond et Putzier, 1964 ; Gomez et al., 1972).

Par ailleurs, on ne peut pas exclure totalement qu'un peu de plutonium de la cicatrice ne finisse par diffuser vers le compartiment systémique mais cela reste, de toutes façons, marginal.

\subsection{Dosimétrie systémique}

\subsubsection{Dosimétrie des émetteurs alpha}

Dans le cas d'espèce, par ajustement à la courbe théorique de Durbin sur les 10 premiers jours en tenant compte du facteur 50 d'action du DTPA, la charge systémique est évaluée à $700 \mathrm{~Bq}$. L'utilisation de la courbe d'excrétion monoexponentielle du complexe Pu-DTPA, sur la même durée, conduit à une valeur de $670 \mathrm{~Bq}$. Par ailleurs, la mesure cumulée de l'excrétion urinaire sous DTPA, obtenue par simple totalisation sur 2 à 3 semaines, est de $600 \mathrm{~Bq}$. On vérifie donc qu'elle est du même ordre de grandeur que la charge systémique, soit 700 ou $670 \mathrm{~Bq}$ selon la méthode de calcul.

L'interprétation conduisant à cette valeur de la charge systémique est corroborée par les résultats des examens urinaires sur le long terme (Fig. 2). Il est en effet prévu que les résultats se situent :

(a) hors administration de DTPA, dans la bande limitée par les deux courbes théoriques tracées à partir du $30^{\mathrm{e}}$ jour ; ces deux courbes reflètent le fait que l'estimation de la charge systémique faite à partir des premières mesures sous DTPA est tributaire de l'efficacité du chélateur qui peut aller de 25 à 100 , soit respectivement un facteur deux en dessous et au-dessus de la valeur nominale de 50 : corrélativement, l'excrétion urinaire hors DTPA attendue sur le moyen et le long terme est elle-même affectée de ce facteur deux d'incertitude, 
(b) en cas d'administration de DTPA, un facteur de 25 à 100 au-dessus de cette même bande, le facteur d'efficacité étant plus proche de 100 pour une injection unique (pic très marqué d'excrétion) ou plus proche de 25 en fin de série pour des injections répétées (épuisement de l'action du DTPA au bout de plusieurs jours).

Les valeurs mesurées sous DTPA correspondent typiquement à la situation (b), et se rapprochent de la bande, ou y sont incluses dans la situation (a) hors DTPA.

Par ailleurs, l'excrétion fécale observée confirme l'estimation de la charge systémique fixée de $700 \mathrm{~Bq}$ (Fig. 3). Les résultats des mesures sont en effet compatibles avec la courbe théorique si l'on tient compte du fait que les variations de l'excrétion fécale sont plus amples et brusques que celles de l'excrétion urinaire et que le DTPA peut favoriser l'élimination du plutonium systémique par voie biliaire et intestinale.

La valeur de $700 \mathrm{~Bq}$ correspond à 25 fois la limite annuelle de dose engagée applicable au personnel professionnellement exposé aux rayonnements ionisants. Rappelons que pour le plutonium, cette limite se rapporte au risque non stochastique à l'os et représente une dose équivalente de $500 \mathrm{mSv}$. Ainsi, la dose engagée sur 50 ans est estimée à environ $12 \mathrm{~Sv}$ pour l'os qui est l'organe-cible principal et à $650 \mathrm{mSv}$ pour la dose effective (Piechowski et al., 1992). En pratique, il faut prendre en compte le fait que ces doses sont réparties sur 50 années et que les doses annuelles vont en décroissant au cours du temps.

\subsubsection{Dosimétrie $d u^{24 I} \mathrm{Pu}$}

En l'absence de résultats de mesure urinaire, l'estimation de la charge systémique en ${ }^{241} \mathrm{Pu}$ ne peut être qu'indicative et doit être considérée comme un simple ordre de grandeur. Elle se fonde sur les résultats du frottis réalisé sur le tournevis contaminé (émetteurs alpha $=42000 \mathrm{~Bq}$ et ${ }^{241} \mathrm{Pu}=930000 \mathrm{~Bq}$ ). Sachant que la charge systémique en émetteurs alpha est de $700 \mathrm{~Bq}$, celle en ${ }^{241} \mathrm{Pu}$ est déduite à partir des résultats du frottis et est supposée égale à $(700 \times 930000) / 42000 \simeq$ $16000 \mathrm{~Bq}(c f . \S 1.1)$. La dose engagée sur 50 ans est estimée à environ $6 \mathrm{~Sv}$ pour l'os et à $300 \mathrm{mSv}$ pour la dose effective. Cela représente 10 fois la limite annuelle de dose.

\subsection{Dosimétrie systémique totale}

Globalement, la dose systémique due à l'ensemble des actinides absorbés et fixés dans l'organisme est de l'ordre de $18 \mathrm{~Sv}$ pour l'os et $950 \mathrm{mSv}$ pour la dose effective. Cet incident a donc conduit à une exposition estimée à 35 fois la limite 
annuelle de dose. Il convient de bien comprendre que cette exposition est étalée dans le temps, en décroissant progressivement sur 50 ans.

\section{Enseignements et éléments de réflexion tirés de ce cas concret}

Il a paru intéressant à propos de ce cas de plaie contaminée de faire ressortir un certain nombre de singularités dans les conséquences pratiques des concepts dosimétriques et d'évaluation de risque :

- l'absence d'une méthode de référence établie par la CIPR, pour l'estimation dosimétrique des plaies contaminées peut être une cause de contestation de l'estimation dosimétrique qui a été réalisée,

- l'association des expositions internes et externes est justifiée dans une démarche épidémiologique d'évaluation de risque pour une population exposée. Par contre elle pose un problème de pertinence et de sens pour une seule personne, problème de sens aggravé par la nécessité de comptabiliser sur un an un équivalent de dose engagée sur 50 ans,

- l'importance de la contamination dans ce cas, induit des conséquences socioprofessionnelles avec un reclassement obligatoire. Ce type de conséquences entraîne des difficultés importantes dans les petites et moyennes entreprises aux activités spécialisées en milieu nucléaire,

- la potentialité de contamination notable, suite à une blessure même bénigne en apparence, doit entraîner une grande vigilance pour les opérations en boîte à gants,

- enfin la complexité des notions à expliquer à propos de la dosimétrie et du risque associé génère des problèmes de communication dans la nécessité légitime d'informer.

\section{RÉFÉRENCES}

Bistline R.W., Watters R.L., Lebel J.L. (1972) A Study of the Translocation Dynamics of Plutonium and Americium from Simulated Puncture Wounds in Beagle Dogs, Health Phys. 22, 829-831.

Carbaugh E.H., Decker W.A., Swint M.J. (1989) Medical and Health Physics Management of a Plutonium Wound, Radiat. Prot. Dosim. 26, 345-349.

CEC, DOE \& IPSN (1995) Guidebook for the treatment of accidental internal radionuclide contamination of workers (G.B. Gerber, R.G. Thomas, Eds.) Radiat. Prot. Dosim., traduction : «Traitement de la contamination interne accidentelle des travailleurs », Institut de protection et de sîreté nucléaire (1995), Fontenay-aux-Roses.

Code du travail :

décret n ${ }^{\circ} 86-1103$ du 02/10/1986,

- décret $n^{\circ}$ 9l-963 du 19/09/1991,

- décret $\mathrm{n}^{\circ} 75-306$ du 28/04/1975,

- décret $n^{\circ} 88-662$ du 06/05/1988,

- arrêté du 28/08/1991 : recommandations aux médecins du travail assurant la surveillance médicale des travailleurs exposés aux rayonnements ionisants. 
Durbin P.W. (1972) Plutonium in Man: a New Look at the Old Data, Radiobiology of Plutonium (B.J. Stover, W.S.S. Jee, Eds.), pp. 469-530. The J.W. Press, Salt Lake City.

Gomez L.S., Lebel J.L., Watters R.L. (1972) The effect of lymph node removal on $\mathrm{PuO}_{2}$ translocation, Health Phys. 22, 833-836.

Hammond S.E., Putzier E.A. (1964) Observed Effects of Plutonium in Wounds over a long Period of Time, Health Phys. 10, 399-406.

Harrison J.D., David A.J., Stather J.W. (1978) Experimental Studies of the Translocation of Plutonium from Simulated Wound Sites in the Rat, Int. J. Radiat. Biol. 33, 457-472.

IAEA (1996) Assessment and treatment of external and internal radionuclide contamination, TECDOC-869, Vienna, Austria.

ICRP Publication 54 (1988) Individual Monitoring for Intakes of Radionuclides by Workers: Design and Interpretation, Ann. ICRP 19(1-3).

ICRP Publication 78 (1998) Individual Monitoring for Internal Exposure of Workers, Ann. ICRP 27(3/4).

Jolly L. Jr, McClearen H.A, Poda G.A., Walke J.P. (1972) Treatment and Evaluation of a Plutonium238 Nitrate Contaminated Puncture Wound - A two-years case history, Health Phys. 23, 333341.

Piechowski J., Cavadore D., Tourte J., Cauquil M.H., Raynaud P., Harduin J.C., Thomas P., Chaptinel Y. (1989) Model and Practical Information concerning the Radiotoxicological Assessment of a Wound Contaminated by Plutonium, Radiat. Prot. Dosim. 26, 265-270.

Piechowski J., Menoux B., Chaptincl Y. (1992) Évaluation de l'exposition systémique résultant d'une blessure contaminée par des produits radioactifs, rapport CEA-R-5583, C.E. Saclay, Gif-surYvette.

Schofield G.B. (1969) Comparison in the Medical Management of Three Cases of Plutonium Contaminated Wounds, IAEA, Vienna.

Schofield G.B., Howells H., Ward F., Lynn J.C., Dolphin G.W. (1974) Assessment and Management of a Plutonium Contaminated Wound Case, Health Phys. 26, 541-554.

Talbot R.J., Newton D., Warner A.J. (1993) Metabolism of Injected Plutonium in Two Healthy Men, Health Phys. 65, 41-46.

Volf V. (1989) Optimisation and status of chelation therapy, Radiat. Prot. Dosim. 26, 331-335. 